\title{
Predictive value of plasma parameters in the risk of postpartum ketosis in dairy cows
}

\author{
Yu Cao, Jiang Zhang, Wei Yang, Cheng Xia, \\ Hong-You Zhang, Yan-Hui Wang, Chuang Xu \\ Department of College of Animal Science and Veterinary Medicine, \\ Heilongjiang Bayi Agricultural University, Daqing 163319, China \\ zhy478@163.com
}

Received: June 13, $2016 \quad$ Accepted: March 6, 2017

\begin{abstract}
Introduction: The predictive value of selected parameters in the risk of ketosis and fatty liver in dairy cows was determined. Material and Methods: In total, 21 control and 17 ketotic Holstein Friesian cows with a $\beta$-hydroxybutyrate (BHBA) concentration of $1.20 \mathrm{mmol} / \mathrm{L}$ as a cut-off point were selected. The risk prediction thresholds for ketosis were determined by receiver operating characteristic (ROC) curve analysis. Results: In the ketosis group, paraoxonase-1 (PON-1) activity and concentration of PON-1 and glucose (GLU) were decreased, and aminotransferase (AST) activity as well as BHBA and non-esterified fatty acid (NEFA) contents were increased. The plasma activity and concentration of PON-1 were significantly positively correlated with the level of plasma GLU. The plasma activity and concentration of PON-1 were significantly negatively correlated with the levels of AST and BHBA. According to ROC curve analysis, warning indexes of ketosis were: plasma PON-1 concentration of $46.79 \mathrm{nmol} / \mathrm{L}$, GLU concentration of $3.04 \mathrm{mmol} / \mathrm{L}$, AST concentration of $100 \mathrm{U} / \mathrm{L}$, and NEFA concentration of $0.82 \mathrm{mmol} / \mathrm{L}$. Conclusion: This study showed that the levels of PON-1, GLU, AST, and NEFA could be used as indicators to predict the risk of ketosis in dairy cows.
\end{abstract}

Keywords: dairy cow, ketosis, plasma parameters, predictive value.

\section{Introduction}

Ketosis is a common serious metabolic disease in the perinatal period. Its main clinical symptoms are ketonaemia, ketonuria, and ketone bodies in milk (17). Negative energy balance is the pathological basis of ketosis. Dry matter intake is reduced during early lactation and the demand for energy, fat, and protein is increased (3), therefore, cows mobilise body fat to meet the lactation needs. This leads to further increases in blood ketone level, re-esterification of excess free fatty acids and their storage in the liver, and development of fatty liver (16). Currently, plasma content of $\beta$-hydroxybutyrate (BHBA; $1.2 \mathrm{mmol} / \mathrm{L}$ ) is internationally recognised as the gold standard for diagnosis of ketosis (11). The gold standard for the diagnosis of fatty liver is hepatic biopsy, but liver fat content must be $>50 \mathrm{mg} / \mathrm{g}$, with obvious clinical symptoms (6). Clinical methods to study hepatic steatosis are limited because they cannot be successfully applied in advance (9). Researchers are looking for an effective and rapid diagnostic method to diagnose fatty liver in dairy cows. Many studies indicate that ketosis, abnormal liver function, and fatty liver of dairy cows are closely related. High incidence of ketosis, high level of plasma BHBA $(>2.50 \mathrm{mmol} / \mathrm{L})$, and high activity of aspartate aminotransferase (AST $>100 \mathrm{U} / \mathrm{L}$ ) lead to incidence of fatty liver in dairy cows (18). Paraoxonase-1 (PON-1), also known as arylesterase, is a calcium-dependent esterase that is synthesised and secreted primarily by the liver. PON-1 plays an important role in antioxidant protection because of its ability to hydrolyse products of lipid peroxidation (8). It has been shown that both are related to the activity of PON-1 and intrahepatic lipid content. The activity of PON-1 is used as a specific marker for diagnosis of fatty liver (5). The aim of this study was to measure blood PON-1 and other selected biochemical indicators that could be used to predict ketosis in dairy cows. 


\section{Material and Methods}

Experimental animals and feed composition. The study was carried out in a dairy herd comprising in total more than 3000 Holstein Friesian cows that were maintained in a free-stall housing using a complete dairy management software system (Afifarm, Israel) in Heilongjiang, China. The average calving interval of the herd was 444 days and the 305-day mean milk yield $23.6 \mathrm{~kg} /$ day. All cows were $2-4$ years old, with parity of 1-3. Twenty-one healthy cows and 17 cows with ketosis were selected randomly, according to the plasma concentration of BHBA $(>1.2 \mathrm{mmol} / \mathrm{L})$ and clinical symptoms. The cows were fed total mixed ration (TMR) at 4:00 a.m. and 4:00 p.m. during the postpartum period. The TMR consisted of corn, concentrated feed, soybean, ensiling, Leymus chinensis, calcium hydrophosphate, baking soda, and salt. Feed analysis displayed $75.6 \%$ of dry matter, $11.65 \%$ of crude protein, $20.32 \mathrm{MJ} / \mathrm{kg}$ of net energy for lactation, $4.1 \%$ of fat, $0.65 \%$ of $\mathrm{Ca}$, and $0.44 \%$ of $\mathrm{P}$.

Sample collection. Blood samples were collected from the vena caudalis mediana using $\mathrm{Na}$ heparin in the early morning, and then immediately centrifuged at $1400 \times \mathrm{g}$ for $10 \mathrm{~min}$ at room temperature. The supernatants were aliquoted into Eppendorf tubes ( $1 \mathrm{~mL}$ plasma/tube) and stored at $-80^{\circ} \mathrm{C}$ until analysis.

Parameters and analytical methods. PON-1 enzyme activity towards paraoxon as a substrate was quantified spectrophotometrically as described by Gan et al. (7). Concentration of plasma PON-1 was assayed using a PON-1 ELISA kit (Haling Biological Technology, China). BHBA was assayed using an ELISA kit (Randox Laboratories, UK). Levels of plasma glucose (GLU), non-esterified fatty acid (NEFA), Ca, P, Mg, and AST were measured directly using an automatic biochemistry analyser.

Statistical analysis. Experimental data were analysed by $t$-test and Pearson correlations, disease was predicted by binary logistic analysis, and diagnostic threshold values were determined by receiver operating characteristic (ROC) curve analysis. Binary logistic analysis was used to establish two simulation models using the above indicators to predict fatty liver in dairy cows. Model I had two indicators of lipid metabolism, GLU and NEFA. Model II had eight indicators of liver metabolism, including PON-1 activity, PON-1 concentration, and AST. Sensitivity, specificity, positive predictive value (PPV), negative predictive value (NPV), positive likelihood ratio $(+\mathrm{LR})$, and negative likelihood ratio (-LR) were calculated in the corresponding $95 \%$ confidence intervals.

\section{Results}

Comparison of blood biochemical indexes in experimental cows. Compared with the control group, plasma PON-1 activity and levels of PON-1 and GLU were significantly decreased in the ketosis group at 1421 days postpartum (Table 1).

Correlation between PON-1 and blood biochemical indexes. To illustrate further the correlation coefficients between plasma PON-1 and other biochemical analytes, Pearson correlation coefficients were calculated on days 14-21 postpartum (Table 2). PON-1 concentration was positively correlated with PON-1 activity and the level of GLU AST, BHBA, and NEFA. PON-1 activity was positively correlated with PON-1 and GLU concentration, and negatively correlated with the activity of AST and the level of BHBA.

Table 3 and Fig. 1 show the sensitivity, specificity, NPV, PPV, +LR, and -LR which were calculated in the corresponding 95\% confidence intervals for warning indexes of ketosis in dairy cows. Critical thresholds were determined by the Youden index, with PON-1 >46.79 nmol/L, GLU >3.04 $\mathrm{mmol} / \mathrm{L}, \mathrm{AST}<100 \mathrm{U} / \mathrm{L}$, and NEFA $<0.82 \mathrm{mmol} / \mathrm{L}$. The sensitivity of PON-1, GLU, AST, and NEFA was $89.47 \%, 89.47 \%, 83.33 \%$, and $64.29 \%$, respectively. The specificity of PON-1, GLU, AST, and NEFA was $100 \%, 86.36 \%, 90 \%$, and $69.57 \%$, respectively. The area under the curve for PON-1, GLU, AST, and NEFA was $0.960,0.952,0.927$, and 0.742 , respectively. Therefore, the activity of AST and concentrations of PON-1, GLU, and NEFA predicted ketosis in dairy cows.

Table 1. Mean ( \pm standard error) and P-value for 38 blood biochemical parameters in the control and ketosis groups

\begin{tabular}{llll}
\hline Parameters & C group $(\mathrm{N}=21)$ & T group $(\mathrm{N}=17)$ & P value \\
\hline PON-1 $(\mathrm{nmol} / \mathrm{L})$ & $52.26 \pm 7.07$ & $43.36 \pm 3.0$ & 0.005 \\
PON-1 $(\mathrm{U} / \mathrm{ml})$ & $85.01 \pm 22.71$ & $70.69 \pm 15.84$ & 0.045 \\
AST $(\mathrm{U} / \mathrm{L})$ & $86.19 \pm 18.12$ & $159.41 \pm 61.44$ & 0.0 \\
$\mathrm{BHBA}(\mathrm{mmol} / \mathrm{L})$ & $0.66 \pm 0.18$ & $3.37 \pm 1.38$ & 0.0 \\
NEFA $(\mathrm{mmol} / \mathrm{L})$ & $0.53 \pm 0.31$ & $0.88 \pm 0.4$ & 0.004 \\
GLU $(\mathrm{mmol} / \mathrm{L})$ & $3.56 \pm 0.56$ & $2.41 \pm 0.6$ & 0.0 \\
$\mathrm{Ca}(\mathrm{mmol} / \mathrm{L})$ & $3.56 \pm 0.56$ & $2.41 \pm 0.6$ & 0.0 \\
$\mathrm{P}(\mathrm{mmol} / \mathrm{L})$ & $1.97 \pm 0.18$ & $1.87 \pm 0.28$ & 0.213 \\
$\mathrm{Mg}(\mathrm{mmol} / \mathrm{L})$ & $1.68 \pm 0.37$ & $1.83 \pm 0.55$ & 0.337 \\
\hline
\end{tabular}

PON-1 - paraoxonase-1; AST - aspartate aminotransferase; BHBA - $\beta$-hydroxybutyric acid; NEFA - nonesterified fatty acid; GLU - glucose 
Table 2. Correlation between PON-1 and plasma biochemical indicators in dairy cows $(\mathrm{N}=38)$

\begin{tabular}{lll}
\hline Parameters & PON-1 content & PON-1 activity \\
\hline PON-1 content & 1 & $0.616^{* *}$, \\
PON-1 activity & $0.616^{* *}$ & 1 \\
AST & $-0.42^{*}$ & $-0.426^{* *}$ \\
BHBA & $-0.595^{* *}$ & $-0.546^{* *}$ \\
NEFA & $-0.595^{* *}$ & 0.336, \\
GLU & $0.453^{* *}$ & $0.537^{* *}$, \\
Ca & 0.198 & -0.024, \\
P & -0.064 & 0.116 \\
$\mathrm{Mg}$ & 0.367 & 0.34 \\
\hline
\end{tabular}

$* \mathrm{P}<0.05$. ** $\mathrm{P}<0.01$

PON-1 - paraoxonase-1; AST - aspartate aminotransferase; BHBA

- $\beta$-hydroxybutyric acid; NEFA - non-esterified fatty acid; GLU - glucose

Table 3. Cut-off point, sensitivity, specificity, NPV, PPV, +LR, -LR, and area under the ROC curve

\begin{tabular}{|c|c|c|c|c|c|c|c|c|}
\hline Parameters & Thresholds & $\begin{array}{l}\text { Sensitivity } \\
(\%)\end{array}$ & $\begin{array}{l}\text { Specificity } \\
(\%)\end{array}$ & $\begin{array}{l}\text { NPV } \\
(\%)\end{array}$ & $\begin{array}{l}\text { PPV } \\
(\%)\end{array}$ & $+\mathrm{LR}$ & -LR & AUC \\
\hline PON-1 & 46.79 & 89.47 & 100 & 90.47 & 100 & 0 & 1.05 & 0.96 \\
\hline GLU & 3.04 & 89.47 & 86.36 & 90.47 & 82.35 & 6.41 & 0.14 & 0.952 \\
\hline AST & 100 & 83.33 & 90 & 85.71 & 88.24 & 8.33 & 0.185 & 0.927 \\
\hline NEFA & 0.82 & 64.29 & 69.57 & 76.19 & 56.25 & 2.11 & 0.51 & 0.742 \\
\hline
\end{tabular}

NPV - negative predictive value; PPV - positive predictive value; LR - likelihood ratio; ROC - receiver operating characteristic; PON-1 - paraoxonase-1; AST - aspartate aminotransferase; BHBA - $\beta$-hydroxybutyric acid; NEFA - nonesterified fatty acid; GLU - glucose

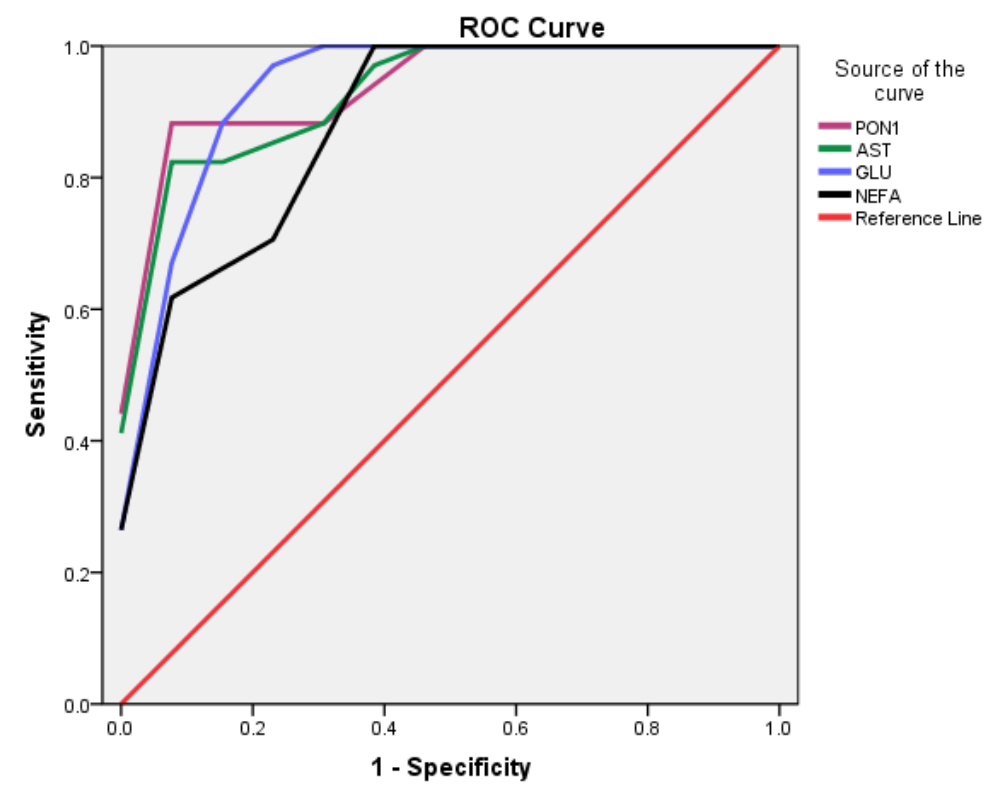

Fig. 1. ROC Curve Receiver operating characteristic (ROC) plots for PON-1, AST, BHBA, NEFA, and GLU for diagnosis of ketosis (PON1 - paraoxonase-1; AST aspartate aminotransferase; BHBA - $\beta$-hydroxybutyric acid; NEFA - non-esterified fatty acid; GLU - glucose)

\section{Discussion}

Liver plays an important role in energy metabolism during the transition period in ruminants. It is the main location of energy metabolism and conversion, and it is important for ensuring the normal production in dairy cows. Ketosis and fatty liver are the major metabolic diseases of dairy cows that can cause liver injury, and pose a serious threat to the health of lactating cows. It is thus necessary to develop an efficient and feasible test to assess the health status of the liver in dairy cows to reduce the losses caused by nutritional metabolic diseases. However, current clinical energy and liver indices are not sufficiently sensitive, specific, and accurate to reflect liver fat deposition (10). Therefore, it is important to find a sensitive index of hepatic lipid deposition to prevent ketosis and fatty liver in dairy cows.

PON-1 is synthesised by the liver and its main physiological function is to participate in the oxidation 
of high-density and low-density lipoproteins (14). Previous studies have indicated that a decrease in PON-1 activity has important diagnostic significance for hepatic fat deposition. The present study showed that, compared with control group, the concentration and activity of plasma PON-1 in fatty liver significantly decreased, and PON-1 was significantly negatively correlated with BHBA content. After calving, due to the large energy output and insufficient energy supply, cows are in a condition of negative energy balance and body lipid mobilisation to meet normal production. The large amount of fatty acids that are produced through body fat mobilisation generates a large number of reactive oxygen species (ROS) during the $\beta$-oxidation process taking place in the mitochondria and endoplasmic reticulum of hepatocytes (15). This excess of ROS and free radical depletion of antioxidant factors leads to disruption of the dynamic balance between oxides and antioxidants, which causes oxidative stress (4). Lipid peroxides can create a large number of cytotoxic intermediates leading to liver cell necrosis and death, and inflammation or inflammatory cell infiltration in the liver parenchyma (1). This leads to a reduction of PON-1 activity when fatty liver occurs. Similarly, we confirmed that the activity and concentration of PON-1 are obviously reduced when fatty liver is present. This might be related to oxidative stress that is inversely proportional to PON-1 activity (2).

Moreover, NEFA has hepatotoxic activity and can enhance toxicity of cytokines, such as tumour necrosis factor, and increase mitochondrial swelling and permeability, liver cell degeneration, necrosis, or apoptosis (12). In the present study, the concentrations of NEFA and PON-1 were negatively correlated, and there was no direct correlation between PON-1 activity and NEFA, which indicates that PON-1 concentration is more sensitive than PON-1 activity in liver lipid deposition. Meanwhile, the activity of AST was significantly increased, and there was a significant negative correlation between PON-1 and AST in our study. AST is present in the cytoplasm or mitochondria of many cell types, especially in the liver, heart, and skeletal muscles. Therefore, AST activity is the highest in these tissues. When fatty liver occurs, cell membrane permeability is increased, enzymes are released into the bloodstream, and the activity of enzymes in the blood becomes increased. Therefore, AST is used as a marker of hepatic lipid deposition in the diagnosis of fatty liver (13).

Ketosis and fatty liver, as nutritional and metabolic diseases, are harmful to the health and, as a consequence, production of animals. Therefore, early diagnosis of ketosis and fatty liver is an effective method of prevention and control of the diseases. In the present study, binary regression analysis and ROC curve analysis were used to establish an early warning model of ketosis and fatty liver; the concentration levels for PON-1 should not be $<46.79 \mathrm{nmol} / \mathrm{L}$, for GLU $<3.04 \mathrm{mmol} / \mathrm{L}$, for AST $>100 \mathrm{U} / \mathrm{mL}$, and for
NEFA $>0.82 \mathrm{mmol} / \mathrm{L}$. If one or more indicators are above or below the standards, it indicates that the cows are at risk of ketosis or fatty liver. Under such circumstances, it is essential to immediately assess diet, body condition, and other feeding habits to ensure adequate energy supply.

In summary, this study demonstrated that concentrations of PON-1, GLU, and NEFA, and activity of AST could be used to predict the risk of ketosis and fatty liver in dairy cows, and the determination of PON-1 content is the most useful.

Conflict of Interests Statement: The authors declare that there is no conflict of interests regarding the publication of this article.

Financial Disclosure Statement: This study was financially supported by grants from the National Science and Technology Support Program, China and National Science and Technology Project "Twelfth Five-Year" in rural areas, National Science Foundation Committee, China Spark Program, and the NCET.

Animal Rights Statement: The study protocol was approved by the Ethics Committee on the Use and Care of Animals of Heilongjiang Bayi Agricultural University (Daqing, China). The study was approved by the farm owner and all animal experiments were conducted according to the International Guiding Principles for Biomedical Research. The protocol was approved by the Committee on the Ethics of Animal Experiments of the Heilongjiang Bayi Agricultural University.

Acknowledgements: The authors would like to thank the International Science Editing.

\section{References}

1. Adams L.A., Lindor K.D.: Nonalcoholic fatty liver disease. Ann Epidemiol 2007, 17, 863-869.

2. Aviram M., Rosenblat M.: Paraoxonases 1, 2, and 3, oxidative stress, and macrophage foam cell formation during atherosclerosis development. Free Radic Biol Med 2004, 37, 1304-1316.

3. Baird G.D.: Primary ketosis in the high-producing dairy cow: clinical and subclinical disorders, treatment, prevention, and outlook. J Dairy Sci 1982, 65, 1-10.

4. Bouwstra R.J., Goselink R.M.A., Dobbelaar P., Nielen M., Newbold J.R., Werven V.T.: The relationship between oxidative damage and vitamin $\mathrm{E}$ concentration in blood, milk, and liver tissue from vitamin E supplemented and nonsupplemented periparturient heifers. J Dairy Sci 2008, 91, 977-987.

5. Farid A.S., Honkawa K., Fath E.M., Nonaka N., Horii Y.: Serum paraoxonase-1 as biomarker for improved diagnosis of fatty liver in dairy cows. BMC Vet Res 2013, 9, 73.

6. Gerloff B.J., Herdt T., Emery R.S.: Relationship of hepatic lipidosis to health and performance in dairy cattle. J Am Vet Med Assoc 1986, 188, 845-850.

7. Gan K.N., Smolen A., Eckerson H.W., Du B.N.L.: Purification of human serum paraoxonase/arylesterase. Evidence for one 
esterase catalyzing both activities. Drug Metab Dispos 1991, 19, 100-106.

8. Kulka M., Bełtowski J., Kluciński W., Orłowska M., Kołodziejska J., Kleczkowski M: Serum paraoxonase-1 activity of dairy Holstein-Fresian cows in different lactation stagespreliminary study. Pol J Vet Sci 2014, 17, 143-147.

9. LeBlanc S.: Monitoring metabolic health of dairy cattle in the transition period. J Reprod Dev 2010, 56, 29-35.

10. Oetzel G.R. Monitoring and testing dairy herds for metabolic disease. Vet Clin North Am Food Anim Pract 2004, 20, 651-674.

11. Padilla L., Shibano K.I., Inoue J., Tohru T., Tohru H.: Plasma vitamin $\mathrm{C}$ concentration is not related to the incidence of ketosis in dairy cows during the early lactation period. Vet Med Sci 2015, 67, 883-886.

12. Pessayre D., Mansouri A., Haouzi D., Fromenty B.: Hepatotoxicity due to mitochondrial dysfunction. Cell Biol Toxicol 1999, 15, 367-373.

13. Seifi H.A., Gorji-Dooz M., Mohri M., Dalir-Naghadeh B., Farzaneh N.: Variations of energy-related biochemical metabolites during transition period in dairy cows. Comp Clin Path 2007, 16, 253-258.

14. Turk R., Juretić D., Gereš D., Svetina A., Turk N., FlegarMeštrić Z.: Influence of oxidative stress and metabolic adaptation on PON-1 activity and MDA level in transition dairy cows. Anim Reprod Sci 2008, 108, 98-106.

15. Videla L.A.: Energy metabolism, thyroid calorigenesis, and oxidative stress: functional and cytotoxic consequences. Redox Rep 2000, 5, 265-275.

16. White H.M.: The Role of TCA cycle anaplerosis in ketosis and fatty liver in periparturient dairy cows. Animals 2015, 5, 793-802.

17. Xu C., Xu Q., Chen Y.Y., Wei Y., Cheng X., Hong Y.Z., Kui L.Z., Tai Y.S., Zi Y.Z.: FGF-21: promising biomarker for detecting ketosis in dairy cows. Vet Res Commun 2016, 40, 47-57.

18. Xu C., Wang Z.: Comparative proteomic analysis of livers from ketotic cows. Vet Res Commun 2008, 32, 263-273. 